\title{
An Adaptive Prediction based Approach for Congestion Estimation in Active Queue Management (APACE)
}

\author{
Abhishek Jain, Abhay Karandikar and Rahul Verma \\ Department of Electrical Engineering, \\ Indian Institute of Technology, Bombay, India
}

\begin{abstract}
Active Queue Management (AQM) policies are mechanisms for congestion avoidance, which pro-actively drop packets in order to provide an early congestion notification to the sources. In this paper we propose a new Adaptive Prediction based Approach for Congestion Estimation in Active Queue Management (APACE) that predicts the instantaneous queue length at a future time using adaptive filtering techniques. We compare the performance of APACE with other existing AQM schemes like RED [1], SRED [2], AVQ [3] and PAQM [4] in networks having both single and multiple bottleneck links. We show that the performance of APACE in terms of the stability of the instantaneous queue is comparable to that of PAQM, which is one of the best AQM strategies. Moreover APACE performs better than PAQM in terms of link utilization and in networks with multiple bottleneck links. APACE is not very sensitive to parameter settings, adapts quickly to changes in traffic and can achieve a given delay or packet loss by varying just one parameter, thus giving the network operator a better tool to manage congestion and improve the performance of the network.
\end{abstract}

Index Terms-Active Queue Management, RED parameters, Congestion Control, Operating Point, APACE.

\section{INTRODUCTION}

Active Queue Management (AQM) policies attempt to estimate the congestion at a node and signal the incipient congestion by dropping packet(s) before the buffer is full. The main aim of the RED [1] scheme was of providing "congestion avoidance" by dropping packets in anticipation of congestion. The performance of the RED algorithm depends significantly upon the setting of each of its parameters, which appears to be a difficult problem. In [5], Hollot et al. have studied the problem of tuning RED parameters from a control theoretic view point. The aim was to improve the throughput by controlling oscillations in the instantaneous queue. Feng et al. [6] proposed a mechanism for adaptively varying one of the RED parameters, $\max _{p}$, with the aim of reducing the packet loss rates across congested links. Floyd et al. in [7] discuss the algorithmic modifications to the self-configuring RED algorithm [6] for tuning $\max _{p}$ adaptively. Despite all these studies, doubts still persist about the useful deployment of RED [8], [9].

To address some of the problems of RED, recently there have been some proposals in active queue management. BRED [10] and FRED [11] aim to improve the fairness of RED by maintaining per-active-flow state information. AVQ [3] tries to decouple congestion measure from the performance measure. Predictive AQM (PAQM) [4] tries to exploit traffic predictability in the calculation of the packet dropping probability.

In this paper, we propose and analyze a new AQM strategy called APACE and compare its performance with exist- ing schemes like RED, AVQ, SRED and PAQM. Our simulation results indicate that the APACE scheme is able to learn the changes in network quickly and thus perform well under fluctuating traffic conditions. In terms of instantaneous queue, the performance of APACE is comparable to that of PAQM, which by far gives the most stable instantaneous queue. Moreover, APACE performs remarkably well by achieving higher link utilization $^{1}$ and a lower packet loss for a given delay ${ }^{2}$ in networks with multiple bottleneck links. Link utilization in APACE remains almost constant at a high value irrespective of its parameter settings, thus enabling the network operator to improve on other performance metrices (such as delay and packet loss) keeping the link utilization high.

The rest of the paper is organized as follows. In Section II we explain the APACE algorithm, discuss its prediction accuracy in Section III, significance of its various parameters in Section IV. We then compare the performance of our scheme (APACE) with RED, SRED, AVQ and PAQM and in Section V for single bottleneck link scenarios and in Section VI for multiple bottleneck link scenarios. We finally conclude in Section VII.

\section{PRoposed SCHEME}

In APACE, we estimate the congestion by predicting the instantaneous queue length at a future time instant. This estimate is based on the queue lengths at the previous packet arrivals. The decision to drop any packet is based on the predicted value of the instantaneous queue length rather than the average queue length, as in the case of RED. As will be shown later in this paper, this makes the scheme more responsive especially in scenarios with changing network conditions. We now explain the APACE scheme in detail.

\section{A. Predicting the Instantaneous Queue}

We predict the instantaneous queue length using the Normalized Least Mean Square (NLMS) algorithm [12]. Our simulation results illustrate that the NLMS predictor can be used to get a good estimate of the instantaneous queue length under a large set of network scenarios (different kinds of sources, topologies etc). Moreover, the algorithm takes only a few iterations to converge and adapts well under changing network scenarios.

The instantaneous queue length prediction is made at every packet arrival. Let $M$ denote the order of the NLMS filter

\footnotetext{
${ }^{1}$ By link utilization, we refer to the number of packets transmitted successfully on the particular link.

${ }^{2}$ By delay, we refer to the average queuing delay.
} 
used for prediction, $q(n)$ the instantaneous queue length at the $n$th packet arrival and $\bar{q}(n)$ a $M \times 1$ vector of the instantaneous queue lengths of the past $M$ packet arrivals. The instantaneous queue length after $N_{0}$ packet arrivals from the $n^{\text {th }}$ packet is predicted based on $\bar{q}(n)$. We call $N_{0}$ the prediction parameter. We denote the predicted queue length by $\hat{q}\left(n+N_{0}\right)$ which is calculated as follows:

$$
\hat{q}\left(n+N_{0}\right)=\bar{w}_{q}^{T}(n) * \bar{q}(n)
$$

In the above equation, $\bar{w}_{q}(n)$ denotes a $M \mathrm{x} 1$ weight vector. These weights are updated dynamically based on the error between the predicted and the actual queue length. The error, $e(n)$ in the prediction is computed as

$$
e(n)=q\left(n+N_{0}\right)-\bar{w}_{q}^{T}(n) * \bar{q}(n)
$$

The queue weights are updated using the following equation:

$$
\bar{w}_{q}(n+1)=\bar{w}_{q}(n)+\mu(n) * \bar{q}(n) * e(n)
$$

In the NLMS algorithm, $\mu(n)$ is calculated using the following equation:

$$
\mu(n)=\frac{\mu_{0}}{1+\bar{q}^{T}(n) \bar{q}(n)}
$$

The queue weights are initially set to fixed values and later updated using the above equations. Typically, the weights are initially set to 0 . The value of $\mu_{0}$ is set to 0.01 .

\section{B. Taking a Packet Drop Decision}

As stated earlier, the decision to drop the incoming packet is based on the predicted value of the instantaneous queue length. The incoming packet is dropped with a probability $p$ that is calculated based on $\hat{q}\left(n+N_{0}\right)$. The algorithm for dropping the incoming packet(s) is illustrated in Figure 1. Let $B$ denote the maximum buffer size. If $\hat{q}\left(n+N_{0}\right)<\alpha * B$, no packet is dropped ( $\alpha$ is a positive constant less than 1). If $\hat{q}\left(n+N_{0}\right)>B$, every incoming packet is dropped. If $\alpha * B \leq \hat{q}\left(n+N_{0}\right) \leq B$, an incoming packet is dropped with a probability $p$, which is a function of the predicted queue size. For the purpose of simulations, we vary the probability $p$ linearly from 0 at $\alpha B$ to $\max _{p}$ at $B$. The motivation behind linearly increasing the packet dropping probability is to make the scheme more aggressive as the predicted queue length increases. The packet dropping probability, $p$ can thus be expressed as:

$$
p \leftarrow \frac{\max _{p}\left(\hat{q}\left(n+N_{0}\right)-\alpha * B\right)}{(1-\alpha) * B}
$$

\section{PREDICTION ACCURACY}

The first issue that needs to be addressed is whether the NLMS algorithm can predict the instantaneous queue length accurately. We test the performance of NLMS algorithm under different network loads. The simulations have been performed using the network simulator, ns v2.1b8a [13]. The network topology shown in Figure 2 has been used for simulations. Figure 2 illustrates the mean square error in the actual

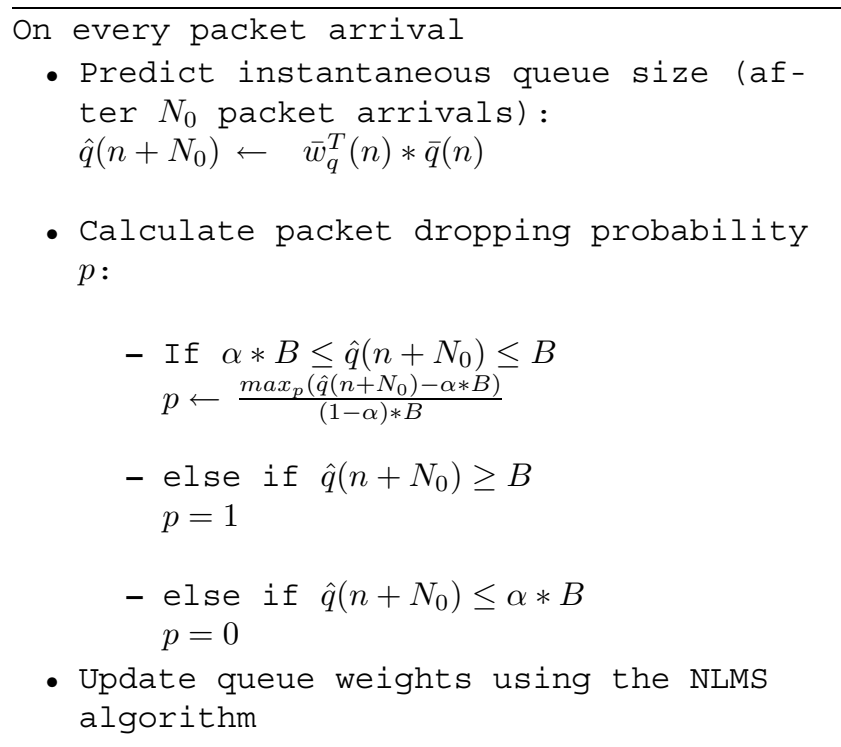

Fig. 1. The APACE algorithm

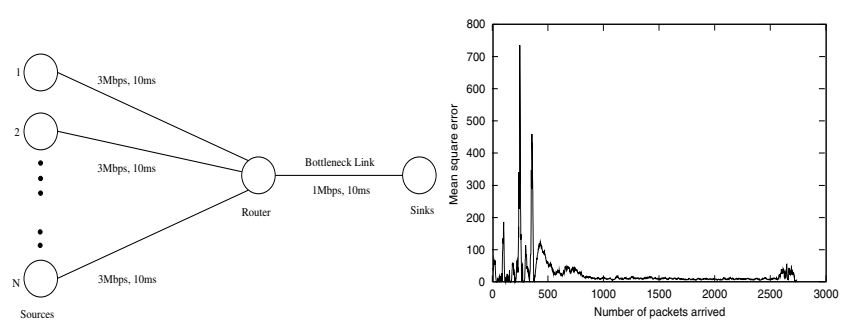

Fig. 2. Network topology and Learning curve

and the predicted value of the instantaneous queue size (learning curve). The plot has been obtained for 25 TCP sources. The packet loss rate is relatively high $\approx(5-10) \%$. The mean square error has been averaged over 100 sample paths. As can be seen from the figure, the NLMS algorithm is able to predict the instantaneous queue to a reasonable accuracy (residual error $^{3} \approx 8$ square packets) and is also able to converge quickly. We get similar learning curves for other network scenarios also, which have not been reported here due to lack of space.

Based on the above discussion and simulation results, we can conclude that the NLMS algorithm does a good prediction of the instantaneous queue.

\section{SIGNIFICANCE OF VARIOUS PARAMETERS}

The various parameters of the scheme are listed in Table I. Our simulation results indicate that the order of the filter, $M$ does not have a significant impact on the performance of the scheme. The algorithm typically takes $20 M$ iterations (packet arrivals) to converge. Moreover, the computation complexity of the scheme increases with increasing $M$. Therefore, it is advisable to take a low value for the order of the filter. We suggest $M \approx(5-20)$ as a good value for the order of the filter and the performance of the scheme is not sensitive to $M$ in this range.

\footnotetext{
${ }^{3}$ By residual error, we refer to the steady state mean square error in predicting
} the instantaneous queue. 
TABLE I

APACE PARAMETERS

\begin{tabular}{|l|l|}
\hline Parameter & Function \\
\hline$M$ & Order of the filter \\
\hline$N_{0}$ & Prediction parameter for the filter \\
\hline axx $_{p}$ & Maximum dropping probability \\
\hline$\alpha$ & $\begin{array}{l}\text { Decides the lower threshold below which } \\
\text { no incoming packet }(\mathrm{s}) \text { is dropped }\end{array}$ \\
\hline
\end{tabular}

The prediction parameter, $N_{0}$ decides the trade-off between the accuracy of the prediction and how early the prediction is made, $i e$, a lower value of $N_{0}$ means that the prediction is made over a relatively shorter time scale. In such a scenario, the prediction error is typically low. On the other hand, by taking a large value of $N_{0}$, the prediction is made over a longer time scale but the prediction error might be large. $N_{0}$ should be large enough so that the effect of dropping a packet results in an early congestion notification to the source.

Our simulation results illustrate that $N_{0}$ too does not have a strong bearing on the performance (in terms of packet loss rate, average queuing delay and link utilization) of the scheme. In this paper we have reported simulation results for $M=10$ and $N_{0}=15$. An extensive simulation study in support of the claims made can be found in [14].

The parameter $\max _{p}$ governs how aggressively the packets are being dropped, based on the predicted value of the instantaneous queue length and $\alpha$ determines the buffer occupancy at which we should start dropping packets. The effects of these parameters on the performance can be found in [14]. We suggest $\max _{p}=0.2, \alpha=0.3, N_{0}=15, M=10$ as the default values for APACE.

\section{Comparison With other Queuing Strategies}

In this section we compare the performance of APACE with other queuing schemes like RED, SRED, PAQM and AVQ. The network topology shown in Figure 2 is used for performing the simulations. The results for multiple bottleneck scenario is explained in the next section. The number of TCP sources, $N$, is varied to achieve different incoming traffic loads. Packet size has been fixed to 500 bytes. The buffer size at the router is of 20 packets for SRED and 50 for all others. The reason for choosing 20 is the fact that SRED always try to keep the buffer close to full. The results (except for the instantaneous queue) have been averaged over 20 sample paths.

\section{A. Instantaneous queue length stability}

We first address the issue of instantaneous queue stability. In our simulation $40 \mathrm{TCP}$ sources are switched on randomly in the first two seconds and the simulation is performed for 40 seconds. Figure 3 illustrates the instantaneous queue size at the gateway for various queuing strategies. We have used $w_{q}=$ $0.002, \min _{t h}=5, \max _{t h}=15, \max _{p}=0.1$ for REDand the default parameters for APACE. The parameters for SRED are $M=1000, \alpha=1 / M$, and $p_{\max }=0.15$. Note that the meanings of $M$ and $\alpha$ are different in SRED. Similarly, the parameters for AVQ are $\gamma=0.98$ and $\alpha=0.10$. For PAQM, $Q_{\text {opt }}=20$ as the queue in APACE is stable at 20 .

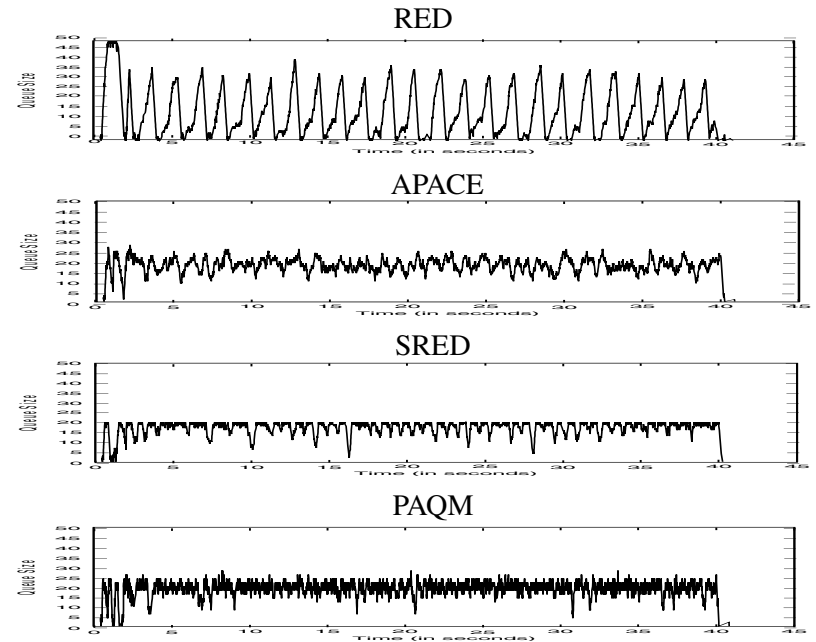

Fig. 3. Instantaneous queue at RED, APACE, SRED and PAQM routers under heavy traffic conditions

As is evident from the plots in Figure 3, RED is unable to control the oscillations in the instantaneous queue, while our scheme (APACE) and PAQM provide reasonable stability to the instantaneous queue. Moreover, even under steady heavy traffic, the instantaneous queue in RED becomes empty frequently (as shown in Figure 3) leading to severe under-utilization of the link.

We next perform the simulation under fluctuating network loads. We switch on 10 sources within a small interval of time. At about 20 seconds from the start of the simulation, 20 new TCP sources are switched on resulting in a sudden increase in the incoming traffic and 10 seconds later 20 of them are switched off. The remaining 10 TCP sources run till 40 seconds from start where the simulation is terminated. The instantaneous queues are kept again around 20 for the sake of comparison and are illustrated in Figure 4. APACE is indeed able to adapt well to the changes in network conditions and in maintaining the stability of the instantaneous queue.
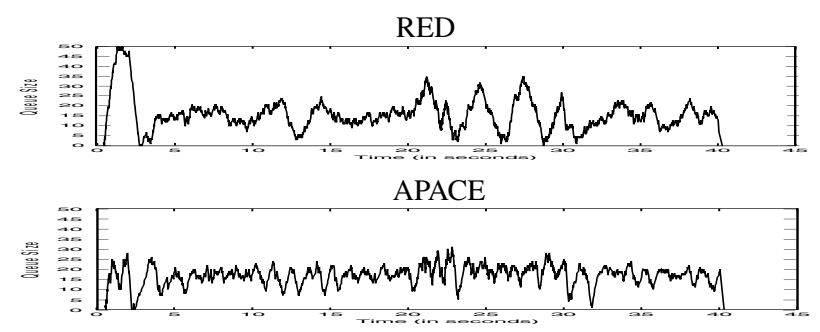

SRED

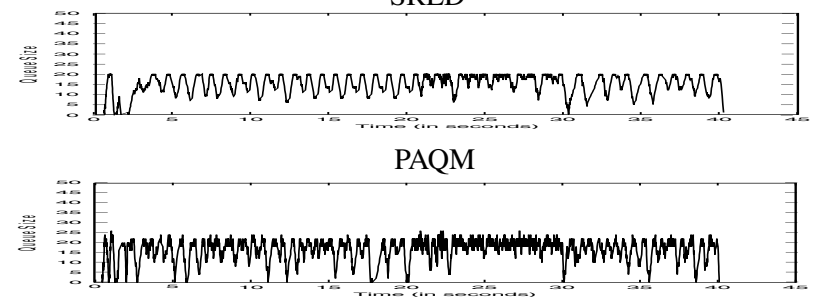

Fig. 4. Instantaneous queue at RED, APACE, SRED and PAQM routers under fluctuating traffic conditions 


\section{B. Link utilization and Packet loss rate}

Figure 5 illustrates the link utilization and fraction of packets lost as the number of TCP connections is increased. As can be seen from the figure, APACE shows a consistent improvement in the link utilization and packet loss rate.

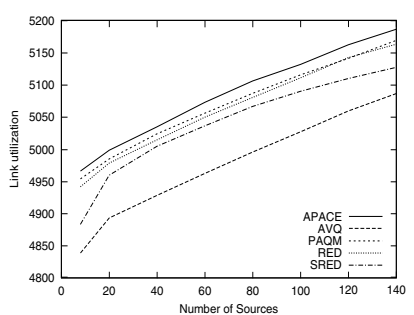

(a)

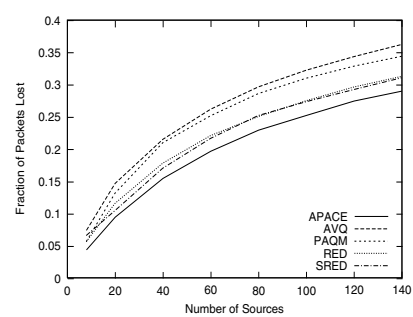

(b)
Fig. 5. Link utilization (a) and fraction of packets lost (b) vs. number of sources in a single bottleneck scenario

\section{Trade-off Comparison with RED}

It should be noted that it is unfair to compare APACE with RED or any other scheme by fixing any one set of parameters as one might achieve an entirely different performance for some other setting of the parameters. Therefore we compare the performance of APACE scheme with RED in terms of the delaylink utilization trade-off curves (refer Figures 6(a), 6(b)) and delay-loss trade-off curves (refer Figures 6(c), 6(d)) that can be achieved. The buffer size is 250 packets. The simulations have been performed with 80 TCP sources. Each curve corresponds

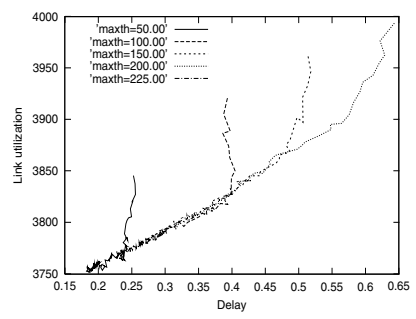

(a)

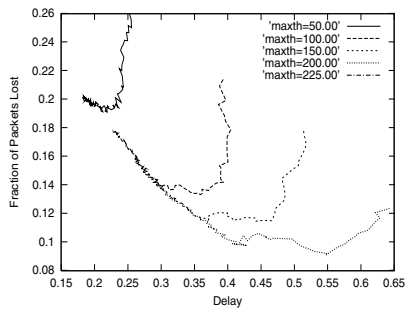

(c)

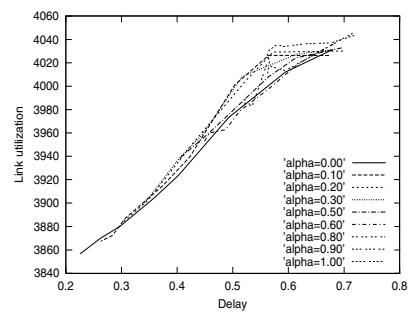

(b)

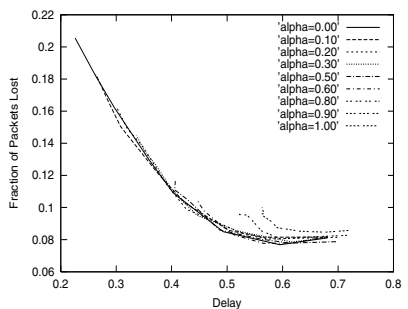

(d)
Fig. 6. Delay-link utilization for RED (a), APACE (b) and Delay-loss for RED (c), APACE (d) trade-off curves for multiple bottleneck scenario

to a different value of $\max _{t h}(\alpha)$ for RED (APACE). The extreme right point on each curve corresponds to $\max _{p}=0$ and as we move along the curve, we get points corresponding to higher values of $\max _{p}$. It is worth noting that the plots for different values of $\alpha$ are close to each other and in particular, the plot with $\alpha=0$, encompasses the complete range of the delaylink utilization trade-off plane covered by the other plots (for different values of $\alpha$ ). Therefore, by setting $\alpha=0$ and varying only $\max _{p}$, one can achieve different delay-link utilization trade-off operating points.

As can be seen from Figures 6(a) and 6(b), APACE gives a better link utilization and also lower delay than RED. Also any operating point that is achievable by RED can be achieved by APACE as well by a suitable setting of parameters. Moreover, for the APACE scheme (refer Figures 6(b), 6(d) one can cover the entire range of operating points by keeping $\alpha=0$ and varying $\max _{p}$ only. The above observations are also true when the buffer size is 50 packets. The same however cannot be said for RED and both the parameters, $\max _{p}$ and $\max _{t h}$ need to be varied in order to achieve a certain operating point. This makes APACE scheme easy to adapt to network conditions. Varying only $\max _{p}$ adaptively based on the network traffic, we can achieve an even better AQM strategy.

We have compared the performance only with RED here, mainly to illustrate the significance of the concept of trade-off curves. Trade-off comparisons with other schemes are reported in the next section for the multiple bottleneck link scenario, which is also more important. From the above we can conclude that the APACE gives a better link utilization with lower delay and only one parameter $\max _{p}$ needs to be varied to achieve any given feasible operating point.

\section{Performance Under Multiple Bottleneck LINKS}

We now compare the performance of the various AQM schemes in networks having multiple congested bottleneck links. The network topology is shown in Figure 7. There are $N$ TCP sources connected to router 1 and a cross traffic from 25 TCP sources flows from router 2 to router 3 and also from router 4 to router 5 similarly. Packet size has been fixed to 500 bytes and buffer size to 50 packets. The results (except for instantaneous queue) have been averaged over 20 sample paths. Routers 2 and 4 are the most congested nodes and hence

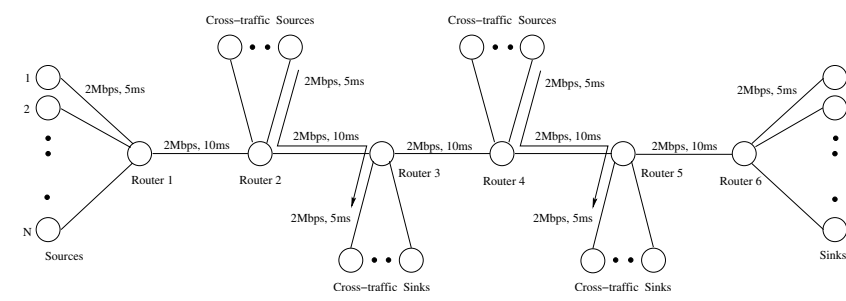

Fig. 7. Network topology for multiple bottleneck links

show similar behavior. We have chosen router 2 for detailed study. We verify our earlier observation, by extensive simulations [14], that values of $N_{0}$ and $M$ do not affect the performance significantly. We choose the same parameter settings as before, except the $Q_{o p t}=40$ for PAQM and a buffer size of 40 packets for SRED at all the five nodes.

\section{A. Instantaneous Queue, Link utilization and Packet loss rate}

The instantaneous queue length results [14] are similar to those obtained for single bottleneck link (the queue occupancy 
is around 40 packets instead of 20 previously), and hence are not reported due to lack of space. Figure 8 illustrates the link

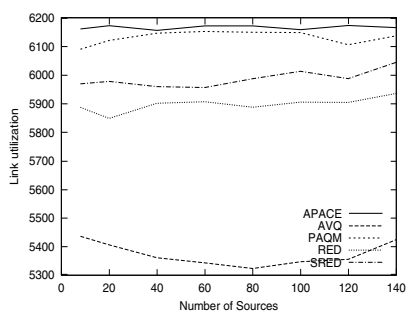

(a)

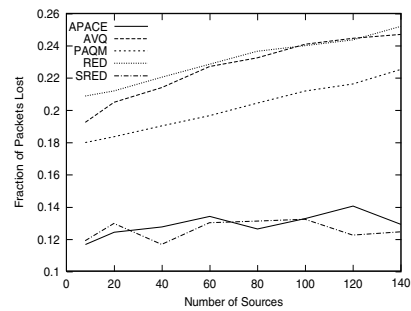

(b)
Fig. 8. Link utilization (a) and fraction of packets lost (b) vs. number of sources in a multiple bottleneck scenario

utilization and fraction of packets lost as the number of TCP connections are increased. We observe that in networks with multiple bottleneck links, APACE is able to achieve higher link utilization with lower packet loss rate and a stable queue similar to PAQM that keeps the delay bounded.

\section{B. Trade-off Curves}

The trade-off curves for the various schemes are shown in Figure 9. For APACE we plot the curves for $\alpha=0$ while comparing it with others to illustrate the fact that we can indeed fix $\alpha$ at 0 or some other small value and vary only $\max _{p}$ to get a good performance. For RED, the curves correspond to $\max _{t h}=15$ and varying $\max _{p} . Q_{o p t}$ in PAQM and buffer size in SRED varies from 3 to 50 as we move from left to right and $\alpha$ varies from 0.05 to 0.99 in AVQ. We observe that for a given delay APACE achieves the highest link utilization at a much lower packet loss rate. Though the trade-off curves for SRED look better, it is associated with other problems like global synchronization owing to the constant overflow of its buffer. In addition, link utilization in APACE remains almost constant at a high value indicating that it is quite independent of its parameter settings and hence the network operator can focus more on the delay and packet loss rate without worrying much about link utilization.

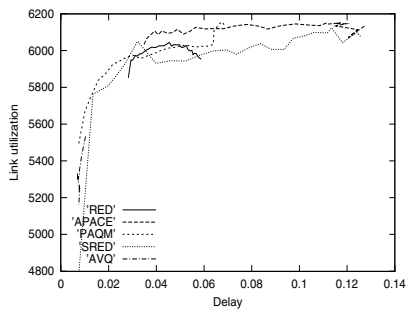

(a)

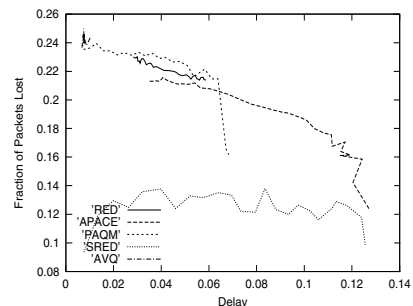

(b)
Fig. 9. Delay-link utilization (a) and Delay-loss (b) trade-off curves for multiple bottleneck scenario

Note that the drop in the link utilization-delay trade-off curve in Figure 9(a) for $\max _{t h}=15$ which is the default value for RED indicates that we would achieve lower link utilization and higher packet delays at least for the traffic scenario considered here (which is common) for higher values of $\max _{p}$. This suggests that varying $\max _{p}$ adaptively may sometimes worsen the performance of RED under multiple bottleneck links.

\section{CONCLUSIONS AND FUTURE WORK}

In this paper, we have presented a novel AQM scheme based on (predicted) instantaneous queue length. We conclude that algorithms like NLMS can indeed be used to predict the instantaneous queue length. The APACE scheme performs better than existing AQM schemes as it adapts faster to changes in network conditions and is able to keep the instantaneous queue stable. APACE also gives higher link utilization and a much lower packet loss rate as compared to PAQM in addition to the instantaneous queue stability comparable to PAQM. In addition the scheme is able to achieve better operating points than that of PAQM and others(in terms of delay-link utilization or delayloss trade-off) for both single and multiple bottleneck links. The link utilization of APACE remains more or less constant at a high value, in networks with multiple bottlenecks, with change in its parameters that can be varied suitably to achieve a given delay and packet loss rate without worrying much about link utilization. Moreover this can be achieved by varying just one of its parameters, $i e, \max _{p}$. APACE with $\max _{p}$ being varied adaptively is expected to give even better performance and is an area of future research.

\section{ACKNOWLEDGMENTS}

The authors would like to acknowledge Mr. Guanghui He for sharing some of the Network Simulator implementations used in [4].

\section{REFERENCES}

[1] S. Floyd and V. Jacobson, "Random Early Detection Gateways for Congestion Avoidance", ACM/IEEE Transactions on Networking, 1(4):397-413, August 1993.

[2] Tenuis J. Ott, T.V. Lakshman, Larry Wong, "SRED: Stabilized RED", Proceedings of IEEE INFOCOM, pp. 1346-1355, March 1999.

[3] Srisankar Kunniyur, R. Srikant, "Analysis and Design of an Adaptive Virtual Queue (AVQ) Algorithm for Active Queue Management", Proceedings of ACM SIGCOMM, August 2001.

[4] Yuan Gao, Guanghui He, and Jennifer C. Hou, "On Exploiting Traffic Predictability in Active Queue Management", Proceedings of IEEE INFOCOM, June 2002.

[5] C. Hollot, Vishal Misra, D. Towsley, and Wei-Bo Gong, "A Control Theoretic Analysis of RED", Proceedings of IEEE INFOCOM, April 2001.

[6] Wu-chang Feng, Dilip D. Kandlur, Debanjan Saha and Kang G. Shin, "A Self-Configuring RED Gateway", Proceedings of IEEE INFOCOM, pp. $1320-1328,1999$.

[7] Sally Floyd, Ramakrishna Gummadi, and Scott Shenker, "Adaptive RED: An Algorithm for Increasing the Robustness of RED's Active Queue Management", http:// www.icir.org/floyd/papers/adaptiveRed.pdf, August 2001.

[8] T. Bonald , M. May and J. Bolot, "Analytic Evaluation of RED Performance", Proceedings of IEEE INFOCOM, pp. 1415-1424, March 2000

[9] M. May, J. Bolot, C. Diot, B. Lyles, "Reasons not to deploy RED", Seventh International Workshop on Quality of Service, IWQoS'99, London, England, June 1999.

[10] F Anjum, and L. Tassiulas, "Fair Bandwidth Sharing among Adaptive and Non-Adaptive Flows in the Internet" Proceedings of IEEE INFOCOM, pp. 1412-1420, March 1999.

[11] Don Ling, Robert Morris, "Dynamics of Random Early Detection" Proceedings of ACM SIGCOMM, 1997

[12] Simon Haykin, "Adaptive Filter Theory", Third Edition, Prentice-Hall, 1996.

[13] "http://www.isi.edu/nsnam/ns/"

[14] Abhishek Jain, "Congestion Management and Bandwidth Allocation for Best Effort Traffic in Packet Switched Networks", Maters Thesis, Department of Eelectrical Engineering, IIT Bombay, 2003. 